dissipation.

Further laboratory and theoretical studies of such features are likely to yield much valuable insight into the general properties of nonlinear waves and eddies, whether or not the nature of the jovian great red spot ultimately becomes apparent.

P.L. READ Geophysical Fluid Dynamics Laboratory, Meteorological Office (21),

Bracknell, Berkshire RG12 2SZ, UK

1. Antipov, S.V., Nezlin, M.V., Snezhkin, E.N. \& Trubnikov, A.S. Nature 323, 238-240 (1986)

2. Read, P.L. \& Hide, R. Nature 302, 126-129 (1983); 308, 45 48 (1984).

3. Prandtl, L. Essentials of Fluid Dynamics (Blackie, Glasgow, 1952).

4. Gill, A.E. Atmospheric-Ocean Dynamics (Academic, New York 1982)

5. Stone, P.H. J. Fluid Mech. 45, 659-671 (1971)

6. Hide, R. \& Mason, P.J. Geophys. astrophys. Fluid Dyn. 10, 121-156 (1978)

7. Mason, P.J. Phil. Trans. R. Soc. A278, 397.445 (1975)

8. Nezlin, M.V. Soviet Astr. Lett. 10, 221-226 (1984); JETP Lett., 34, 77-80 (1981)

9. Read, P. L. Icarus 65, 304-334 (1986)

10. Maxworthy, T. \& Redekopp, L.G. Icarus 29, 261-271 (1976).

11. Williams, G.P. \& Yamagata, T. J. atmos. Sci. 41, 453-478 (1984).

12. Williams, G.P. J. atmos. Sci. 42, 1237-1243 (1985)

\section{Glycine-binding sites and NMDA receptors in brain}

SIR-I was fascinated by the report by Johnson and Ascher ${ }^{1}$ in which they describe an interaction between the aminoacid neurotransmitters glycine and glutamate in cultured mouse neurons. Glycine is generally accepted as an inhibitory transmitter, certainly within the spinal cord, whereas glutamate is an excitatory transmitter. The effect of glycine, they report, was not blocked by strychnine, which antagonizes the $\mathrm{Cl}$ - mediated inhibitory responses to glycine.

Their data provide a rationale for an observation I made after my colleagues and I published on the presence and distribution of strychnine-insensitive ${ }^{3} \mathrm{H}$-glycine binding sites in supraspinal regions of the rat brain observed using autoradiography ${ }^{2}$. Comparison of our data with the findings of Monaghan and Cotman ${ }^{3}$ on the binding of ${ }^{3} \mathrm{H}$-glutamate to $N$-methyl-Daspartate (NMDA) sites showed that the distributions of glycine and NMDA binding were identical. This is readily demonstrated by comparing Fig. 1 from ref. 2 with Fig. 3 from ref. 3 . The autoradiograms are very similar, perhaps most strikingly so in the hippocampus, where the greatest density of both NMDA and glycine sites is present in the $\mathrm{CA} 1$ radiatum and oriens layers. The only apparent discrepancy in the hippocampus occurs in the stratum lucidum which was labelled by ${ }^{3} \mathrm{H}$-glutamate but not by ${ }^{3} \mathrm{H}$-glycine. However, these glutamate binding sites are kainatesensitive and not NMDA sites ${ }^{3}$. The similarity between glycine and NMDA extends to the distribution of sites labelled by the non-competitive NMDA antagonist ${ }^{3} \mathrm{H}-\mathrm{MK} 801$ (ref. 4).

Thus the effect of glycine, as suggested by Johnson and Ascher, may be mediated through receptors located externally on neuronal membranes and associated with the NMDA receptor. The autoradiographic data certainly support the view that the NMDA receptor complex includes modulatory sites analogous to those in the $\mathrm{GABA}_{\mathrm{A}}$ receptor complex. Interestingly, the modulatory sites previously described in the NMDA receptor complex seem to have endogenous substrates within the brain, namely $\mathrm{Mg}^{2+}$ (ref. 5) and alpha-endopsychosin 6 . Presumably we can now add glycine to this list.

$$
\text { N. G. Bowery }
$$

Neuroscience Research Centre,

Merck Sharp and Dohme,

Terlings Park, Harlow,

Essex CM20 2QR, UK

1. Johnson, J. W. \& Ascher, P. Nature 325, 529-531 (1987) . Bristow, D. R., Bowery, N. G. \& Woodruff, G. N. Eur. J. Pharmac. 126, 303-308 (1986)

3. Monaghan, D. T. \& Cotman, C. W. J. Neurosci. 5, 2909 2919 (1985).

4. Bowery, N. G. \& Hudson, A. L. Br. J. Pharmac. 89, 775P (1986)

Nowak, L., Bregestovski, P., Ascher, P., Herbert, A. \& Prochiantz, A. Nature 307, 462-465 (1984)

. Quirion, R. et al. Peptides 15, 967-973 (1984).

\section{Can a negative quantity be deemed a probability?}

SIR-One could say that it is reasonable to use the idea of negative probabilities ${ }^{1-4}$ if a body of results valid for normal (that is, positive) probabilities is changed in an elegant or informative way when some of the probabilities involved are given negative values. It is for this sort of reason that one introduced negative energy states, negative temperatures and made similar extensions of concepts previously regarded as constrained to have positive numerical values. Hence, although the frequency definition of probability seems to rule out negative probabilities, there could nevertheless be rules obeyed by probabilities in physics or in probability theory itself, which could make it elegant or convenient to use the extended definition. Suggestions for this approach have been made and have in the main been based on quantum theory. The purpose of this letter is to add to the recent discussion ${ }^{1-4}$ by giving a class of simple examples based on thermodynamics.

I have shown elsewhere ${ }^{5}$ that the first and second law of thermodynamics applied to two identical bodies of constant heat capacity, $C$, lead one to the wellknown inequality for the means

$$
1 / 2\left(T_{1}+T_{2}\right) \geqslant\left(T_{1} T_{2}\right)^{1 / 2}
$$

where $T_{1}, T_{2}$ are the temperatures of the two bodies prior to equilibration. Equilibrium by contact yields the left-hand side of
(1) as the final temperature. Equilibration by running a reversible Carnot engine between the bodies yields the right-hand side as the final temperature. Thus we have, reasonably, that constant-entropy equilibration yields a lower temperature than constant energy equilibration. If the bodies have unequal heat capacities $\left(C_{1}\right.$, $C_{2}$ say), then the same procedure leads to the inequality for the weighted means

$$
p T_{1}+q T_{2} \geqslant T_{1}^{p} T_{2}^{q} \quad(p+q=1)
$$

where $p$ and $q$ are formally probabilities, whose interpretation is brought about by the physical argument based on the first and second law of thermodynamics:

$$
p=\frac{C_{1}}{C_{1}+C_{2}}, q=\frac{C_{2}}{C_{1}+C_{2}}
$$

The result (2) is correct mathematically provided the condition $p, q>0$ is added. The positivity and normalization makes $p$ and $q$ normal probabilities.

Next, apply the first and second law to two bodies of different constant heat capacities $C_{2}<0<\mathrm{C}_{1}$, noting that negative heat capacities occur for black holes, stars and other gravitationally bound systems. We could for simplicity consider the case of small temperature changes so that the assumption of constant (temperatureindependent) heat capacities is reasonable. One now finds

$$
p T_{1}+q T_{2} \leqslant T_{1}^{p} T_{2}^{q} \quad(p+q=1)
$$

If $C_{1}+C_{2}<0$ we have $p<0<q$, while $q<$ $0<p$ if $C_{1}+C_{2}>0$. If $T_{1}=2, T_{2}=3$ then an example for the former case is provided by $C_{1}=1, C_{2}=-2$ leading to $4<9 / 2$, and for the latter case by $C_{1}=2, C_{2}=-1$ leading to $1<4 / 3$.

Just as in (2) one can proceed by abstraction from a physical result to a mathematical result, so one can proceed similarly with respect to (4). One arrives at the 'reversed' inequality (4) for means ${ }^{6}$ which are now weighted by one positive and one negative 'probability'. The introduction of negative probabilities is of course not essential even in this case, but at least it does not seem to be too artificial, because of the simple analogy between (2) and (4). We shall explore this matter in a more general setting elsewhere?

\section{P.T. LANDSBERG}

Faculty of Mathematical Studies,

University of Southampton,

Southampton SO9 $5 \mathrm{NH}, \mathrm{UK}$

\footnotetext{
Mückenheim. W. Phys. Rep. 133, 337 (1986)

Maddox, J. Nature 320, 481 (1986)

Parry, D.E. Nature 321, 644 (1986)

Mückenheim, W. Nature 324. 307 (1986)

Landsberg, P.T. Phys. Lett. 67A 1 (1978); 78A, 29 (1980); J. Math. Anal. Appl. 76, 209 (1980).

6. Pečarić, J. Glas. Mat. (Zagreb) Ser. Ill 16, 229 (1981); 19 235 (1984).
}

Landsberg, P.T. \& Pečarić, J. Phys. Rev. (in the press). 